\title{
The Circumstellar Environment of Embedded Massive Stars
}

\author{
Lee G. Mundy, Friedrich Wyrowski, and Sarah Watt
}

Astronomy Department, University of Maryland, College Park, MD 20742

\section{Introduction}

Millimeter and submillimeter wavelength images of massive star-forming regions are uncovering the natal material distribution and revealing the complexities of their circumstellar environments on size scales from parsecs to 100's of AU. Progress in these areas has been slower than for low-mass stars because massive stars are more distant, and because they are gregarious siblings with different evolutionary stages that can co-exist even within a core. Nevertheless, observational goals for the near future include the characterization of an early evolutionary sequence for massive stars, determination if the accretion process and formation sequence for massive stars is similar to that of low-mass stars, and understanding of the role of triggering events in massive star formation.

Two of the most intriguing results from studies of high-mass star formation regions have been the discovery of hot molecular cores (e.g. Olmi et al. 1996, Cesaroni et al. 1994, Walmsley 1995) and the clustering of massive stars. Hot molecular cores are identified by their emission in unusual molecules such as $\mathrm{CH}_{3} \mathrm{CN}$, and by the high brightness temperatures of lines (typically $150-250 \mathrm{~K}$ ) associated with abundant molecules. It is argued that these cores are themselves the sites of new massive star formation (cf. Walmsley 1995). As such, they may represent an important stage in the formation of massive stars. The second result, the clustering of massive stars, suggests that massive stars form in a locally special environment; the structure of their parent cores provides insights into this clustering. The following two sections look at these points more closely.

\section{Hot Cores: Chemistry or Cores?}

Are all regions which show "hot core chemistry" and "hot core emission" massive cores in the act of forming stars? It has often been found that hot core chemistry (Millar et al. 1997, Charnley et al. 1992) is associated with massive, dense cores: masses of 100's to 1000's of solar masses and densities of $10^{7}$ to $10^{8} \mathrm{~cm}^{-3}$ (Walmsley 1995). However, the original prototype for hot cores, the Orion Hot Core, is estimated to be under ten solar masses and the individual hot core tracer molecules have different morphologies when mapped at high resolution (Blake et al. 1996). Is the presence of "hot core emission" truly an indicator of a massive internally heated core or is it simply an indicator of high temperature gas-phase chemistry?

We have recently acquired sub-arcsecond resolution observations of two wellknown hot core sources, G34.26+0.15 and G31.41+0.31, with the objective of 

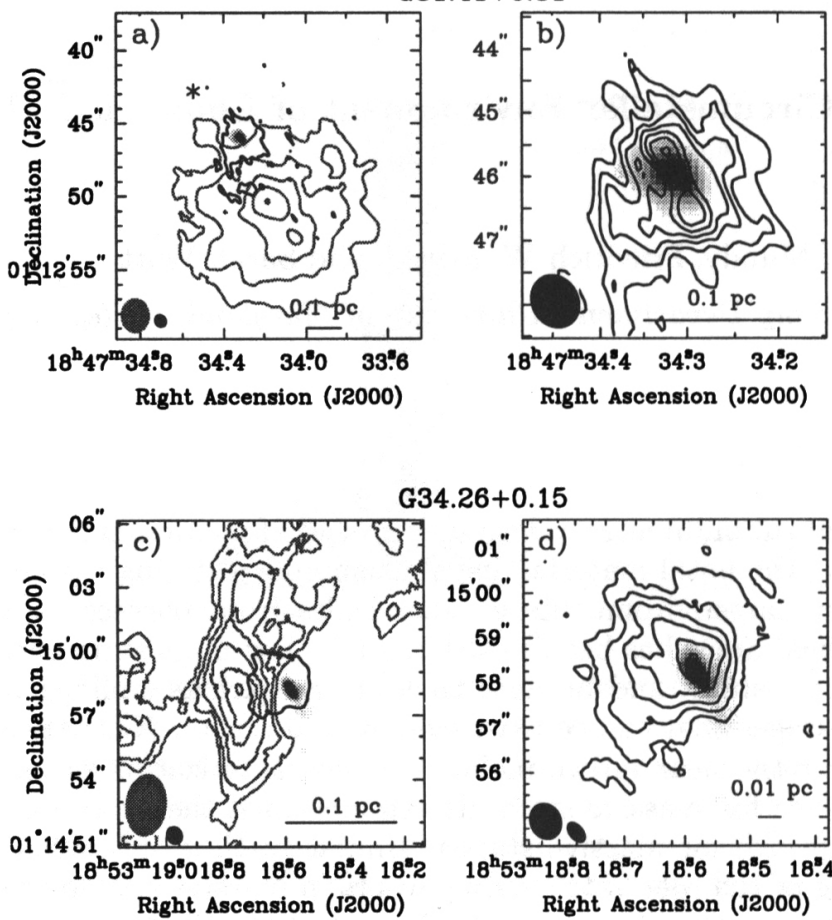

G34.26+0.15

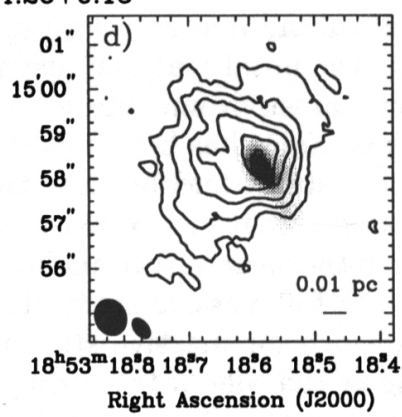

Figure 1. $\lambda=2.7 \mathrm{~mm}$ Continuum, $\mathrm{C}^{18} \mathrm{O}$ and $\mathrm{CH}_{3} \mathrm{CN}$ images for G31.41+0.31 and G34.26+0.15. Panels $a$ and $c$ show the $\mathrm{C}^{18} \mathrm{O}$ emission (grey contours) overlayed on the continuum emission. The dark lines are $4 \sigma$ contours of the $\mathrm{K}=3 \mathrm{CH}_{3} \mathrm{CN}$ emission. Panels $b$ and $d$ show the $\mathrm{J}=6-5 \mathrm{~K}=3 \mathrm{CH}_{3} \mathrm{CN}$ (contours) and continuum (greyscale) emission. The black/white central contour in G31.41+0.31 is a dip.

answering this question. Figure 1 shows maps of the $\lambda=2.7 \mathrm{~mm}$ continuum, $\mathrm{C}^{18} \mathrm{O} \mathrm{J}=1-0$, and $\mathrm{CH}_{3} \mathrm{CN}$ emission from these two regions.

These images are discussed in more detail by Watt et al. (2000). The primary point here is that the $\mathrm{CH}_{3} \mathrm{CN}$ emission, a traditional hot core tracer, is not closely associated with dust continuum emission in G34.26+0.15 (the observed $\lambda=2.7 \mathrm{~mm}$ continuum is consistent with arising entirely from ionized gas in the UC HII region). In G31.41+0.31, the $\mathrm{CH}_{3} \mathrm{CN}$ emission is roughly centered on the continuum emission, and, in this source, there is no compact ionized gas component so the $\lambda=2.7 \mathrm{~mm}$ emission is tracing dust.

Thus, G34.26+0.15 is a hot core without a massive compact core while G31.41+0.31 is a hot core with a massive compact core. We argued previously (Watt and Mundy 1999) that G34.26+0.15 is an example of externally driven hot core chemistry; the current higher resolution images support that picture. The presence of traditional hot core molecules and emission is a statement about the chemical state of the gas: the dust grain mantles have been evaporated and the released molecules are being chemically processed in a hot $(150-300 \mathrm{~K})$ gas environment. This can occur within a dense massive core, or it can occur along the surface of a core which is strongly heated by nearby massive stars. 


\section{The Origin and Structure of Cores: Insights into Cluster Star Formation?}

Recent improvements in the sensitivity and resolution of continuum observations are providing new insights into the structure of massive cores and have stimulated renewed searches for the earliest stages of massive star formation. The Holy Grail is the establishment of an evolutionary sequence for massive stars with observational prototypes. Works by Hunter et al. (2000), Sridharan et al. (1999), and Wyrowski et al. (2000) have provided new candidate high-mass protostellar regions and the work by Hatchell et al. (2000) illustrates the type of structure studies that can be done once these regions are identified. It is clear that forming massive stars are associated with massive cores which typically extend out to scales of order a parsec. The cores contain much more mass than needed for a single star, typically $10^{3}$ to $10^{4}$ solar masses. This is in agreement with the historical wisdom and with the observations that visible massive stars are normally associated with a cluster of lower mass stars.

Interferometric observations able to follow the structure of these cores down to the $10^{3} \mathrm{AU}$ scale. In two sources that we have recently studied, G31.41+0.31 and G10.47+0.31, we find that the power-law density structure of the cores extends from the parsec scale down to around $3000 \mathrm{AU}$. Within roughly 3000 $\mathrm{AU}$, the core structure, as traced by the continuum emission, flattens. This flattening of the density structure in the central core may trace the size scale of the forming cluster. This size scale would likely be set by the angular momentum content of the original cloud, by strength of the original magnetic field, or a combination of the two. This type of scenario where the core feeds material into a central stellar nursery naturally produces star clusters due to gravitational interactions decoupling the protostars from the natal envelope as they grow in mass.

Acknowledgments. We thank the Hat Creek staff for their efforts. We thank R. Cesaroni, M. Walmsley, and E. Churchwell for enjoyable and informative conversations.

\section{References}

Blake, G.A. et al. 1996, ApJ, 472, L49

Cesaroni, R. Churchwell, E., Hofner, P., Walmsley, C.M., \& Kurtz, S. 1994, A\&A, 288, 903

Charnley, S.B., Tielens, A.G.G.M., \& Millar, T.J. 1992, ApJ, 399, 71

Hatchell, J. et al. 2000, A\&A, 357, 637

Hunter, T.R. et al. 2000, AJ, 119, 2711

Olmi, L., Cesaroni, R., Neri, R., \& Walmsley, C.M. 1996, A\&A, 315, 565

Sridharan, T.K. et al. 1999, Star Formation 1999, Nagoya, Japan, Editor: T. Nakamoto, 183

Walmsley, C.M. 1995, Rev. Mex. Astron. Astrof., 1, 137

Watt, S. Mundy, L.G., \& Wyrowski, F. 2000, ApJ, submitted 